\title{
Factor Analysis based on RoboCup Midfielder Position*
}

\author{
Zekai CHENG, Liang NIE, Wenwen JIN, Zhaolong LING, Feng QIN \\ Anhui University of Technology, Ma Anshan, China
}

\begin{abstract}
Research on midfield position has an important meaning for the result of RoboCup Simulation 2D. This paper divides the whole region into eight parts, then count the cycle's number of the both player 6,7 , 8 midfielders in the game in the eight regional positions. Next, three common factors were extracted, which were named as midfielder factor, restricted factor and border defense factor. Finally calculate three factors' scores and get the comprehensive evaluation scores. It is found that the top few teams get good results in recent RoboCup World Cup. It confirms the reasonableness of the factor analysis method.
\end{abstract}

KEYWORD: RoboCup simulation 2D; Factor analysis; Log files; Comprehensive evaluation scores

\section{INTRODUCTION}

Simulation 2D group games run in the environment of a standard computer, using Client/Server mode, competition both sides between Client and Server through the UDP/IP protocol to communicate information[1-3]. To realize players best decisions in the team of Wright Eagle in University of science and technology of China use the action driven of the Markov Decision Process and reveal in sequence, build Markov Transition Matrix[4-6].

Factor Analysis is a kind of common factors extracted from the variable group of statistical techniques, the earliest proposed by the British psychologist Charles Spearman in 1904[7]. Wei-kun LIAO, guo-liang CAI, wen-tao TU[8] using factor analysis method evaluate the level of economic development of the cities in jiangsu province in 2002; Lun RAN, jin-lin LI[9] and others using the factor analysis method on 22 listed companies to evaluate the comprehensive performance; Xue-min WANG[10], faced those listed companies in the Shanghai market in 604 financial statements of the ten main financial indicators for factor analysis in 2001, then according to the situation of each factor score of the stock, has made a comprehensive evaluation.

\section{FACTOR ANALYSIS}

\subsection{Factor analysis principle}

Factor analysis is a multivariate statistical analysis method that concentrate on the study of the relationship among variables, it can transform the variables with complex relationship into a fewer integrated variables.

\subsection{Factor analysis model}

Step 1 Defining the experimental sample variables. Its matrix is shown as expression (1):

$$
X=\left[\begin{array}{lccr}
x_{11} & x_{12} & \ldots & x_{1 \mathrm{p}} \\
x_{21} & x_{22} & \ldots & x_{2 p} \\
\vdots & \vdots & & \vdots \\
x_{n 1} & x_{n 2} & \ldots & x_{n p}
\end{array}\right]
$$

Where $n$ is the times of experimental sample, $p$ is the number of variables to be analyzed. Each row of the matrix represents a group of experimental data, the nth row means the nth group experimental data.

Step 2 Standardizing the data, let the mean value of data be zero and the variance of data be one, then the processed data follows Gaussian distribution. For expressing simple, the standardized matrix is marked as $X$.

\footnotetext{
* Fund Supported: Major projects supported by natural science in Anhui province in 2014(KJ2014ZD05).
} 
The correlation coefficient matrix is shown as expression (2):

$$
A=X^{\prime} X
$$

Supposing the matrix $A$ has eigenvalue $\lambda_{1}, \lambda_{2}, \ldots, \lambda_{p}$, and the corresponding orthogonal eigenvectors are shows as the matrix (3).

$$
V=\left[\begin{array}{lccr}
v_{11} & v_{12} & \ldots & v_{1 \mathrm{p}} \\
v_{21} & v_{22} & \ldots & v_{2 p} \\
\vdots & \vdots & & \vdots \\
v_{p 1} & v_{p 2} & \ldots & v_{p p}
\end{array}\right]
$$

Step 3 Find the component matrix $b_{i j}$ by the method of PCA in this paper despite of so many other methods. The formula is shown as expression (4).

$$
b_{i j}=v_{i j} \cdot \sqrt{\lambda_{j}}
$$

Where $b_{i j}$ is the component of the $i$ th variable on the $j$ th common factor.

Take the common factor matrix as $F$, then

$$
F=V X^{\prime}
$$

Where $X^{\prime}$ is the transposed matrix of $X$.

It is not difficult to draw a conclusion.

$$
F F^{\prime}=\operatorname{diag}\left(\lambda_{1}, \lambda_{2}, \cdots, \lambda_{p}\right)
$$

In the expression (6), $\operatorname{diag}\left(\lambda_{1}, \lambda_{2}, \cdots, \lambda_{p}\right)$ represents this matrix which is a diagonal matrix and the diagonal elements are $\lambda_{1}, \lambda_{2}, \cdots, \lambda_{p}$ respectively, and $F_{i}=V_{i} X^{\prime}, \mathrm{i}=1,2,3, \ldots \mathrm{p}$, that is , $F_{i}$ is the factor score of the $i$ th group experimental sample. Now choose $m(\mathrm{~m}<\mathrm{p})$ main factors, and extract the first main factor from the correlation matrix between the variables, and drown as $F_{1}$, and let the common factor $F_{1}$ have a biggest variance proportion in all the variables. Then, the effect of $F_{1}$ could be ignored; Next, choose the factor $F_{2}$ which is not relate to $F_{1}$ from correlation matrix. And so on, it is not until the communality of all variables could be resolved finished.

Finally, the model is shown as expression (7).

$$
\left\{\begin{array}{l}
X_{1}=b_{11} F_{1}+b_{12} F_{2}+\cdots+b_{1 m} F_{m}+\varepsilon_{1} \\
X_{2}=b_{21} F_{1}+b_{22} F_{2}+\cdots+b_{2 m} F_{m}+\varepsilon_{2} \\
\vdots \\
X_{\mathrm{p}}=b_{p 1} F_{1}+b_{p 2} F_{2}+\cdots+b_{p m} F_{m}+\varepsilon_{p}
\end{array}\right.
$$

The residual $\varepsilon_{1}, \varepsilon_{2} \ldots, \varepsilon_{p}$ are special factors. It should be ignored.

\subsection{Calculating the scores of factors}

Because the common factors could reflect the correlation of original variables, and make these original variables expressed as a linear combination of the common factor. If these factors are expressed as a linear combination of original variables, just as the expression (8) shows.

$$
\left\{\begin{array}{l}
F_{1}=\beta_{11} X_{1}+\beta_{12} X_{2}+\cdots+\beta_{1 p} X_{p} \\
F_{2}=\beta_{21} X_{1}+\beta_{22} X_{2}+\cdots+\beta_{2 p} X_{p} \\
\vdots \\
F_{m}=\beta_{m 1} X_{1}+\beta_{m 2} X_{2}+\cdots+\beta_{m p} X_{p}
\end{array}\right.
$$

\subsection{Comprehensive evaluation scores}

Take each factor variance contribution rate as weighting, and use factor rotation method, combining the variance contribution rate after factor rotation and these factor extracted constitute the linear equations. Finally establish a comprehensive evaluation index function, as shown in type (9):

$$
F_{\text {total }}=\frac{\alpha_{1} F+\alpha_{2} F_{2}+\cdots+\alpha_{m} F_{m}}{\alpha_{1}+\alpha_{2}+\cdots+\alpha_{m}}
$$

Where $\alpha_{i}$ is the contributing rate of variance of $i$ th common factor before rotating or after rotating.

\section{EXPERIMENT}

\subsection{Data modeling}

\subsubsection{Obtaining log files}

In RoboCup simulation 2D game, the server generates $\log$ files, RCG and RCL files. The RCG files records the state of ground in each period, including the ball's coordinate, ball's speed and the coordinate and stamina of all agents, and so on. The RCL files records some command information, etc. The experiment data in this paper is obtained from analyzing $\log$ files.

\subsubsection{Choosing variables}

The field is divided into eight regions, just as figure1 shows.

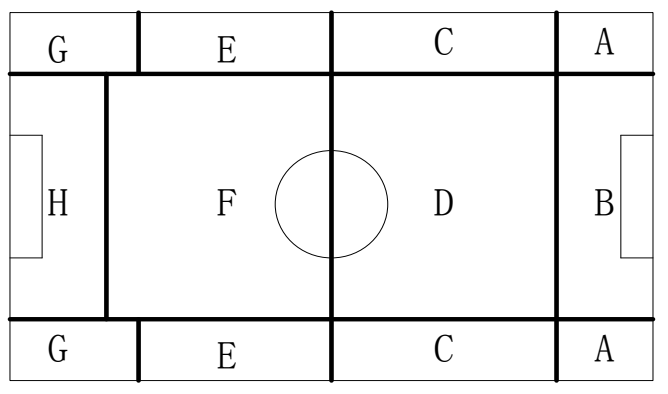

Figure 1. The division of field

Now analyzing the data of player 6 , player 7 , 
player 8 in one game randomly. As is shown in Table 1.

Table1. Data of midfield player(unit: cycles)

\begin{tabular}{|c|c|c|c|c|c|c|}
\hline \multirow{2}{*}{} & \multicolumn{3}{|c|}{ Team1 Player number } & \multicolumn{3}{c|}{ Team2 Player number } \\
\cline { 2 - 7 } & 6 & 7 & 8 & 6 & 7 & 8 \\
\hline $\mathrm{X}_{1}$ & 0 & 127 & 64 & 0 & 36 & 0 \\
\hline $\mathrm{X}_{2}$ & 0 & 163 & 123 & 0 & 6 & 42 \\
\hline $\mathrm{X}_{3}$ & 197 & 377 & 1400 & 3 & 1028 & 1006 \\
\hline $\mathrm{X}_{4}$ & 2497 & 3111 & 2077 & 2648 & 1712 & 1474 \\
\hline $\mathrm{X}_{5}$ & 406 & 117 & 1079 & 0 & 533 & 529 \\
\hline $\mathrm{X}_{6}$ & 2263 & 2108 & 1131 & 2848 & 2147 & 2382 \\
\hline $\mathrm{X}_{7}$ & 328 & 0 & 118 & 0 & 372 & 112 \\
\hline $\mathrm{X}_{8}$ & 308 & 0 & 6 & 499 & 175 & 453 \\
\hline
\end{tabular}

\subsection{Data analysis}

Taking 60 games' log files as experiment data, the results of experiment $\mathrm{KMO}$ and Bartlett Test are shown in table 2.

The result of table 2 shows that the KMO statistics equals 0.721 which is greater than 0.7 .

Table 2. KMO and Bartlett Test

\begin{tabular}{|c|c|c|}
\hline \multicolumn{2}{|c|}{$\begin{array}{c}\text { Kaiser-Meyer-Olkin Measure of Sampling } \\
\text { Adequacy }\end{array}$} & .721 \\
\hline $\begin{array}{c}\text { Bartlett's test of } \\
\text { Sphericity }\end{array}$ & Approx. Chi-Square & 763.309 \\
\hline \multicolumn{2}{|c|}{$\begin{array}{c}\mathrm{df} \\
\text { Sig. }\end{array}$} & 28 \\
\cline { 2 - 3 }
\end{tabular}

The contributing rate of variance and cumulative contribution of variance in each common factor is shown as figure 2 . From the figure, the eigenvalue of three common factors are 3.226,1.724,1.087, respectively, the cumulative contribution of three common factors is $75.453 \%$.

\begin{tabular}{|l|l|r|r|}
\hline \multirow{2}{*}{} & \multicolumn{3}{|c|}{ Component } \\
\cline { 2 - 4 }$\times 1$ & 1 & \multicolumn{1}{|c|}{2} & \multicolumn{1}{c|}{} \\
\hline$\times 2$ & .234 & .813 & .009 \\
$\times 3$ & .070 & .860 & -.048 \\
$\times 4$ & .865 & .100 & -.007 \\
$\times 5$ & .736 & .213 & -.524 \\
$\times 6$ & .087 & .032 & .816 \\
$\times 7$ & -.969 & -.079 & -.098 \\
$\times 8$ & -.228 & -.420 & .631 \\
& .025 & -.707 & .447 \\
\hline
\end{tabular}

Figure 2. Factor loading matrix after rotation

\subsection{Factor rotation}

From figure 2, the correlation between $F_{1}$ and $X_{3}, X_{4}, X_{6}$ is higher, and named midfielder factor.
The correlation between $F_{2}$ and $X_{2}, X_{8}$ is higher, and named restricted area factor. The correlation between $F_{3}$ and $X_{5}, X_{7}$ is higher, and named border defense factor. These three factors could reflect clearly the significance of common factor which is found through the method of factor analysis, and table 3 shows the results.

Table 3. Common factor named

\begin{tabular}{|c|c|c|}
\hline Common factor & Explained variables & Named \\
\hline $\mathrm{F}_{1}$ & $\mathrm{X}_{3}, \mathrm{X}_{4}, \mathrm{X}_{6}$ & Midfielder factor \\
\hline $\mathrm{F}_{2}$ & $\mathrm{X}_{1}, \mathrm{X}_{2}, \mathrm{X}_{8}$ & Restricted area factor \\
\hline $\mathrm{F}_{3}$ & $\mathrm{X}_{5}, \mathrm{X}_{7}$ & Border defense factor \\
\hline
\end{tabular}

\subsection{The score of factors}

Using regression method to find factor score function and common factor and linear relationship between the original variables is shown as type(10):

$$
\begin{aligned}
F_{1}= & 0.008 X_{1}-0.078 X_{2}+0.393 X_{3} \\
+ & 0.291 X_{4}+0.08 X_{5}-0.449 X_{6} \\
- & 0.021 X_{7}+0.124 X_{8} \\
F_{2}= & 0.445 X_{1}+0.479 X_{2}-0.052 X_{3} \\
& -0.09 X_{4}+0.196 X_{5}+0.053 X_{6} \\
& -0.07 X_{7}-0.311 X_{8} \\
F_{3}= & 0.207 X_{1}+0.165 X_{2}+0.063 X_{3} \\
& -0.311 X_{4}+0.633 X_{5}-0.143 X_{6} \\
& +0.371 X_{7}+0.178 X_{8}
\end{aligned}
$$

\subsection{Comprehensive evaluation score}

By taking factor's variance contribution after rotation as weighting, then establish comprehensive evaluation index and extract the common factor $F_{1}, F_{2}, F_{3}$. The linear relation is shown as type(11):

$$
\begin{aligned}
F= & 0.2943 F_{1}+0.26746 F_{2} \\
& +0.19363 F_{3}
\end{aligned}
$$

By this formula, calculate the 60 sets of original data's comprehensive evaluation scores. Now the scores of the top five largest are as shown in figure3.

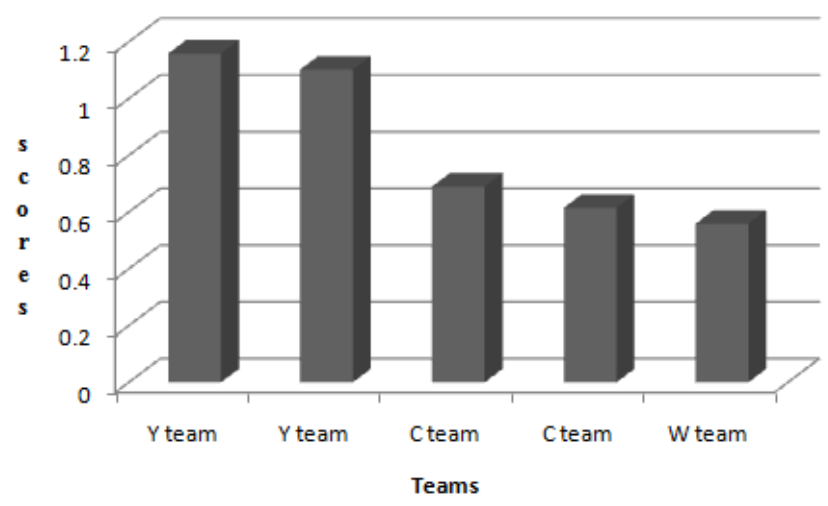

Figure 3. Comprehensive Evaluation Score 


\subsection{Results}

Concluded from the combinaiton of theory and practise, the comprehensive evaluation scores of the three factors including midfielder factor, restricted area factor, border defensive factor that are extracted from the midfielder position factor analysis in Robocup Simulation 2D, can reflect the strength of the team to a certain extent.

\section{CONCLUSION}

In this paper, the method of factor analysis is applied to Robocup Simulation for the first time. Using the thinking of data analysis and data mining to analyse the midfield position with factor analysis, we can extract three common factors including midfield, restricted area and border defense, which can reflect the conclusion of each simulation team strength to a certain extent.

\section{REFERENCES}

[1] Peter, Stone. Veloso M. Multiagent Systems: A survey from a Machine Learning Perspective. Automous Robots, 2000, 8(3):345-383.
[2] Guo Ye-jun, Xiong rong. RoboCup robot soccer simulation game development and design. Computer Engineering and Applications, 2003, 29:146-149.

[3] Yang Yu-pu, Li Xiao-meng, Xu Xiao-zing. A survey of technology of multi-agent cooperation. Information and Control, 2001, 30(4):337-342.

[4] Shi ke. Research on agent decision problems Markov Decision Process Theory. Master's thesis in University of Science and Technology of China 2010.

[5] Feng Wu, Shlomo Zilberstein, Xiaoping Chen. Online Planning for Multi-Agent Systems with Bounded Communication, Artificial Intelligence, 2011, 175(2): 487-511.

[6] Pan ling-han, Chu wei, Cheng xian-yi. RoboCup Soccer strategies based on role. Computer Engineering and Applications, 2004, 26: 66-69.

[7] Su Li-yun, Chen CaiXia, Gao Hong-xia. SPSS19 application of statistical analysis and case-based tutorial. Beijing Hope Electronic Press, 2012.

[8] Liao Wei-kun, Cai Guo-liang, Tu Wen-tao. Urban Economic Development Evaluation Based On Factor Analysis. Statistics and Decision, 2005.

[9] Ran lun, Li Jin-lin. Factor Analysis in the SME sector companies listed on the consolidated results of the evaluation of the application. Mathematical Statistics and Management, 2005, 24 (1): 75-79.

[10] Wang Xue-min. Factor analysis on the evaluation of the stock. Mathematical Statistics and Management, 2004, 23 (3):6-10. 